\title{
In situ measurement of ECM rheology and microheterogeneity in embedded and overlaid 3D pancreatic tumor stroma co-cultures via passive particle tracking
}

\author{
Dustin P. Jones, William Hanna, Gwendolyn M. Cramer \\ and Jonathan P. Celli* \\ Department of Physics \\ University of Massachusetts Boston \\ Boston, MA 02215, USA \\ *Jonathan.Celli@umb.edu
}

Received 29 June 2017

Accepted 18 August 2017

Published 21 September 2017

\begin{abstract}
Tumor growth is regulated by a diverse set of extracellular influences, including paracrine crosstalk with stromal partners, and biophysical interactions with surrounding cells and tissues. Studies elucidating the role of physical force and the mechanical properties of the extracellular matrix (ECM) itself as regulators of tumor growth and invasion have been greatly catalyzed by the use of in vitro three-dimensional (3D) tumor models. These systems provide the ability to systematically isolate, manipulate, and evaluate impact of stromal components and extracellular mechanics in a platform that is both conducive to imaging and biologically relevant. However, recognizing that mechanoregulatory crosstalk is bi-directional and fully utilizing these models requires complementary methods for in situ measurements of the local mechanical environment. Here, in 3D tumor/fibroblast co-culture models of pancreatic cancer, a disease characterized by its prominent stromal involvement, we evaluate the use of particle-tracking microrheology to probe dynamic mechanical changes. Using videos of fluorescently labeled polystyrene microspheres embedded in collagen I ECM, we measure spatiotemporal changes in the Brownian motion of probes to report local ECM shear modulus and microheterogeneity. This approach reveals stiffening of collagen in fibroblast co-cultures relative to cultures with cancer cells only, which exhibit degraded ECM with heterogeneous microstructure. We further show that these effects are dependent on culture geometry with contrasting behavior for embedded and overlay cultures. In addition to potential application to screening stroma-targeted therapeutics, this work also provides insight into how the composition and plating geometry impact the mechanical properties of 3D cell cultures that are increasingly widely used in cancer biology.
\end{abstract}

Keywords: Microrheology; 3D tumor models; matrix remodeling; mechanobiology; stromal depletion.

${ }^{*}$ Corresponding author.

This is an Open Access article published by World Scientific Publishing Company. It is distributed under the terms of the Creative Commons Attribution 4.0 (CC-BY) License. Further distribution of this work is permitted, provided the original work is properly cited. 


\section{Introduction}

The tumor microenvironment encompasses a diverse set of growth-regulating signals that include biochemical and biophysical interactions with stromal cells and surrounding tissues. ${ }^{1-4}$ A key component of this milieu is the extracellular matrix (ECM), a protein-based scaffolding that provides a support framework for healthy tissues, but which takes on altered composition and function as a regulator of tumor growth behavior. Increased production and crosslinking of ECM proteins, and proliferation of cancer-associated stromal fibroblasts are characteristics of the desmoplastic reaction associated with development of many solid tumors. $^{5-7}$ This mechanical microenvironment has been shown to play prominent and complex roles in both constraining tumor growth as well as promoting malignant behavior and invasive progression. ${ }^{3,8-10}$ This stromal involvement is particularly prominent in pancreatic ductal adenocarcinoma (PDAC) noted for development of exceptionally dense, ECM-rich fibrous stroma. ${ }^{5,11-13}$

The study of mechanical regulation of tumor growth by stromal components has been facilitated in large part by the development of in vitro 3D tumor models, which have emerged as a powerful research platform in cancer biology in general. ${ }^{14-18}$ The recreation of $3 \mathrm{D}$ tissue architecture in these model systems provides the ability to systematically isolate and evaluate roles of extracellular mechanical cues and tissue organization that are absent in traditional monolayer cell cultures yet more accessible to imaging than animal tumor models. ${ }^{19,20}$ 3D tumor models utilizing physically customized scaffolds have already been used to gain insight into the effects of matrix composition and rheology impact on integrin and growth factor mediated signaling, and cell motility. ${ }^{21,22}$

At the same time, however, it is important to recognize that interactions involving cancer cells and their mechanical microenvironment are bi-directional. Tumors are not just influenced by physical surroundings, but play an active role in mechanical remodeling; both through pro-fibrotic signals that increase mechanical rigidity as well as through proteolytic breakdown of ECM which needed to achieve invasive motility. ${ }^{23}$ Such modification of the mechanical environment in turn feeds back upon tumor growth as part of two-way biophysical crosstalk between cancer cells and their environment. In order to study these dynamic interactions, it is useful not only to be able to manipulate the extracellular environment in 3D tumor models, but also to be able to monitor the local rheology and microstructure, in situ, without terminating the sample.

In this context, the present study evaluates the potential role of particle-tracking microrheology (PTM) as a method to provide in situ, nondestructive and longitudinal measurements within $3 \mathrm{D}$ co-culture models. ${ }^{24,25}$ Passive PTM uses analysis of the Brownian trajectories of inert, fluorescent tracer probes, embedded during sample preparation as local reporters of a specimen's microstructure and mechanics. Video data, which can be obtained at multiple timepoints and positions within a specimen can be analyzed to estimate the local frequencydependent viscoelastic response through the application of the generalized Stokes-Einstein relation (GSER). ${ }^{26}$ The ability of PTM to perform nondestructive and noncontact measurements on live cell cultures, set this technique apart from many forms of elastography, which requires the sample to come in contact with a physical actuator. ${ }^{27}$ PTM's use of fluorescent tracer probes allows some versatility in resolving measurements within the sample as opposed to other methods, such as AFM, which inherently probe sample surfaces from outside. ${ }^{28}$ PTM has been shown to be useful in measuring tumor level physical interactions, and can be easily collected over a grid in order to inform on the whole sample rheology. ${ }^{29}$ This contrasts with certain active techniques such as optical tweezers which are typically used for studying single cell mechanics and making point measurements within a sample. ${ }^{30}$ Leveraging the relative ease of PTM to acquire data over relatively large spatial scales (trading off microscope field of view and magnification), this approach can also report intra sample microheterogeneity, obtained through statistical analysis of tracer probe trajectories. ${ }^{24}$ Here, building on previous studies establishing the utility of particle tracking measurements for nondestructive longitudinal characterization of ECM remodeling in 3D tumor models, ${ }^{29,31}$ we specifically evaluate the capability of this approach to measure the impact 3D PDAC co-culture composition (with and without fibroblasts) and geometry on the stiffness and microstructure of collagen I ECM. By embedding polystyrene microspheres in the ECM hydrogel at the time of culture preparation, short video 
sequences can be acquired nondestructively while cultures are removed from incubation for brief durations. Using GSER to analyze such data, ECM rigidity of each culture condition at multiple time points reveals as expected a significant increase in ECM shear modulus when stromal fibroblasts are present. Raw particle data are also processed using clustering algorithms to show that fibroblast presence correlates with uniformity and integrity of the ECM. We further investigate the dependence of ECM rheology on sample geometry, comparing PTM measurements in commonly used embedded and overlay $3 \mathrm{D}$ culture geometries.

\section{Materials and Methods}

Pancreatic cancer PANC-1 cells and MRC-5 cells from American Type Culture Collection (ATCC, Rockville, MD) were grown according to ATCC descriptions. DMEM culture media and fetal bovine serum were obtained from Hyclone Laboratories (Logan, UT). All media were supplemented with $50 \mathrm{IU} / \mathrm{mL}$ penicillin and $50 \mathrm{mg} / \mathrm{mL}$ streptomycin (Hyclone Laboratories).

3D cell cultures with embedded probes were prepared by mixing $\mathrm{NaOH}$-neutralized Bovine type I collagen (Corning Inc., Corning, NY) with desired

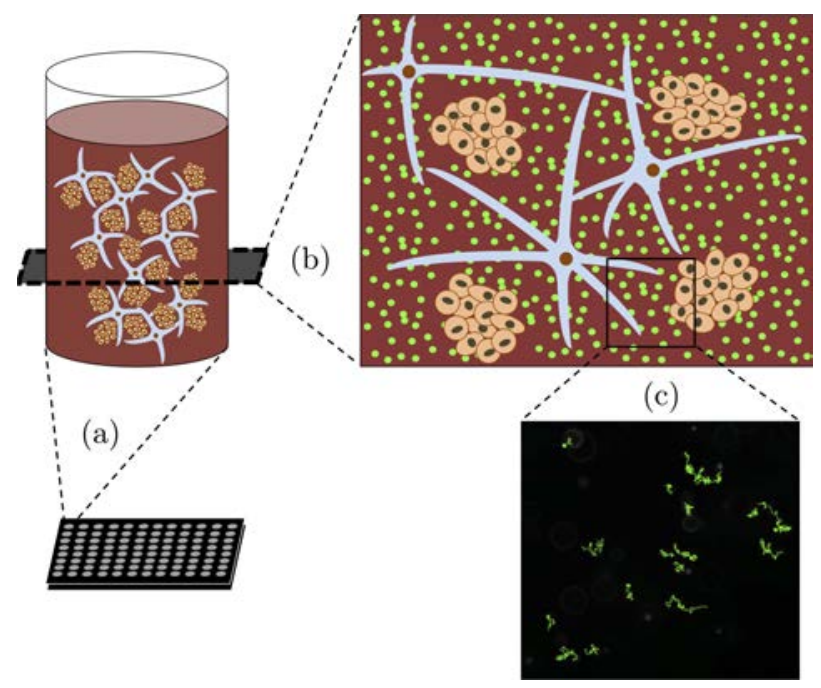

Fig. 1. Schematic representation of PTM applied to a 3D cancer model. (a) 3D cultures containing embedded fluorescent probes are prepared in a 96-well plate. (b) Each well is imaged under $40 \times$ magnification at a uniform $Z$ position. (c) Brownian motion of tracer probes is recorded under fluorescent excitation and video is processed in order to track probe trajectories and calculate MSD. cell suspensions and $1 \mathrm{um}$ diameter yellow-green fluorescent carboxylate modified polystyrene tracer probes (Thermo Fisher Scientific, Carlsbad, CA) at a final stock dilution of 1:250 (or approximately $10^{8}$ microspheres per $\mathrm{mL}$ ) to obtain $1 \mathrm{mg} / \mathrm{mL}$ collagen and initial cell concentrations of 112 cells $/ \mu \mathrm{L}$ for PANC1 and 56 cells $/ \mu \mathrm{L}$ for MRC-5. Certain conditions required embedding of one or both cell types inside the 3D matrix (Fig. 2) while other conditions left the matrix cell-free. Additional culture geometries were produced by overlaying PANC1 cells to the top one set of cell-free matrices and one set of matrices populated with only MRC-5 cells. For all conditions, the final ECM/cell suspension mixture was placed to cure overnight in four replicate wells ( $63 \mu \mathrm{L}$ each) of a 96 well plate for each treatment. Once cured, $30 \mu \mathrm{L}$ of either plain DMEM or appropriate cell suspension was added to the top to hydrate each sample.

Videos were recorded using a Zeiss AxioObserver inverted fluorescence microscope with AxioCam HSm cooled CCD camera (Carl Zeiss Microscopy, Jena, Germany). Three consistently positioned video

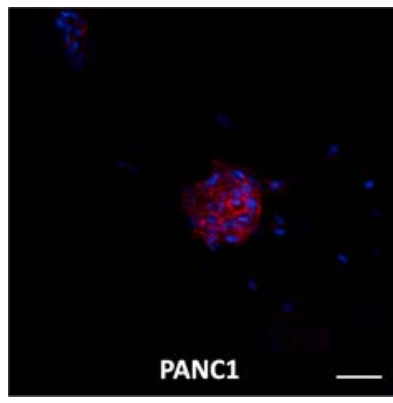

(a)

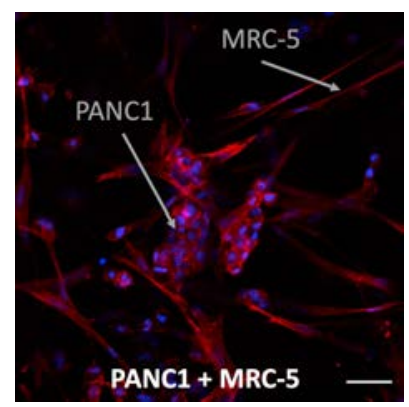

(b)

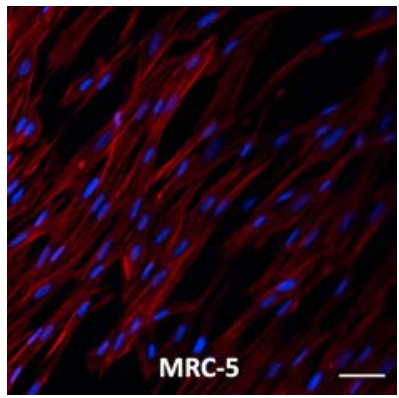

(c)

Fig. 2. Confocal images of (a) PANC1, (b) PANC1 and MRC-5 co-culture, and (c) MRC-5 3D cultures. Nuclei are stained with DAPI (blue) and actin is stained with phalloidin (red). Scale bars $50 u \mathrm{~m}$. 


\section{P. Jones et al.}

locations were mapped onto each well using proprietary Zeiss software. Videos were recorded at approximately $30 \%$ sample height. Video data were analyzed using a methodology for PTM in multiwell $3 \mathrm{D}$ cultures described previously. ${ }^{29}$ This analysis is based on open source particle-tracking routines from the laboratory of Maria Kilfoil. ${ }^{32}$ These routines make it possible to track mean squared displacement (MSD) of fluorescent tracer probes throughout all frames of respective video data and then apply the GSER in order to extract frequency-dependent viscoelastic moduli. Additional statistical analysis of probe trajectories was performed usage the open source clustering function, Mclust, designed for usage with $R$ studio. ${ }^{33}$ For our clustering analysis, we chose a model-based method in which Mclust models the input data for multiple sets of parameters and ranks these models based on their maximum log-likelihood. Ultimately, the best fitting model was a two-component ellipsoidal Gaussian finite mixture model from which a clear threshold displacement value was derived and used to classify probe trajectories.

\section{Results}

The averaged MSDs of tracked probes were compared across three distinct culture conditions: PANC1 homoculture (fibroblast $(-)$ ), PANC1/ MRC-5 fibroblast co-culture (fibroblast $(+)$ ), and cell-free collagen (no cell control). MSDs of probes tracked in fibroblast (-) wells were significantly greater and more heterogeneous than the displacements of probes in control and fibroblast $(+)$ wells (Fig. 3). In fibroblast (-) wells, the overall MSD

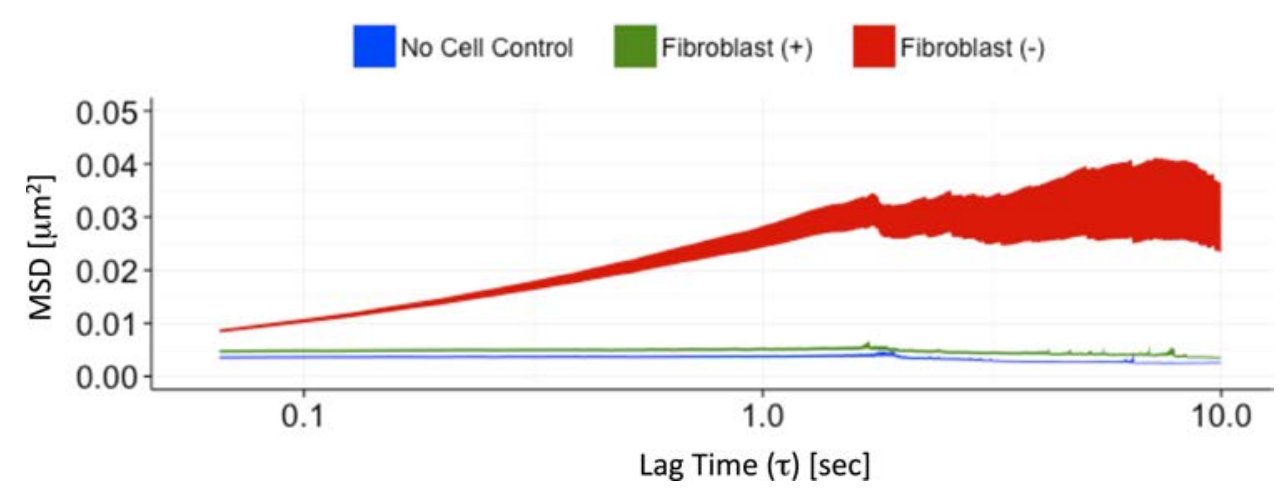

(a)

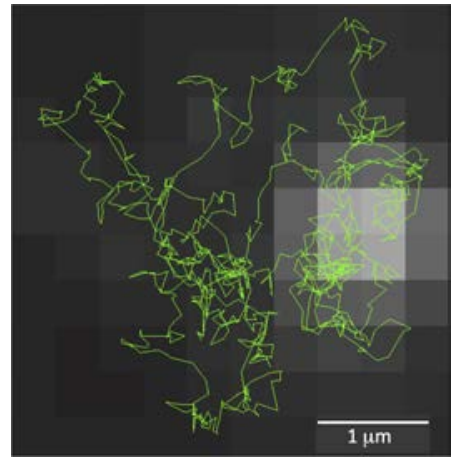

(b)

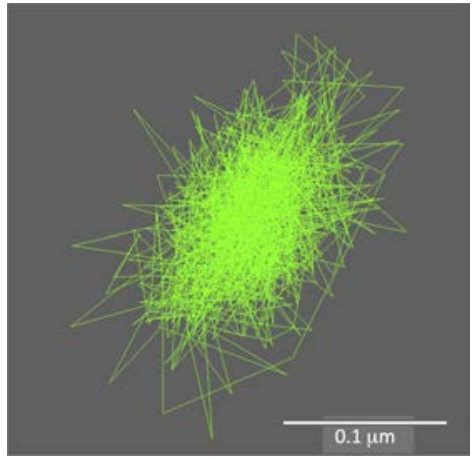

(c)

Fig. 3. (Color online) (a) MSD vs log lag-time for three populations of tracer probes, embedded in either PANC1 homoculture, PANC1 and MRC-5 coculture, or a cell-free control matrix. Probes tracked inside PANC1 cultures exhibited a much greater range of MSDs than those embedded in either of the other conditions, resulting in a steadily amplified shadow of uncertainty with increasing lag time. Generally, probe trajectories fell into two distinct categories: those with (b) "uncaged" trajectory and (c) "caged" trajectory. These two categories of probes can also be seen when plotting a histogram of log maximum displacement, revealing (d) a bimodal distribution of probe trajectories for the fibroblast (-) condition. 


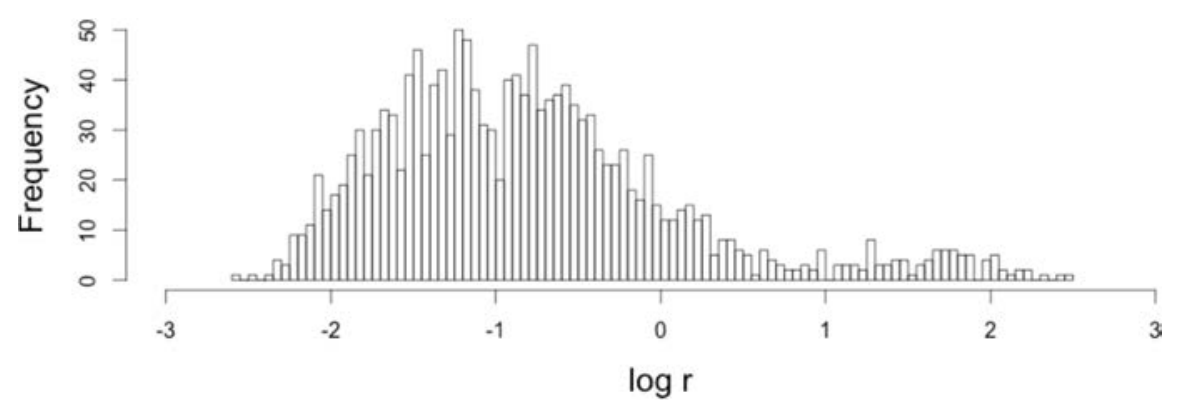

(d)

Fig. 3. (Continued)

reflects averaging over two qualitatively different trajectories; populations of probes with more typical diffusive Brownian motion, and those which appear to be tightly caged within pores in the ECM (representative trajectories in Figs. 3(b) and 3(c), respectively). In fibroblast $(+)$ and cell-free collagen ECM, probe trajectories are more nearly uniformly consistent with the latter description, also reflected in the small MSD over all times probed (Fig. 3(a)). These data suggest that probes in the fibroblast (-) condition encounter a wide distribution of pore sizes relative to the much tighter and more uniform matrix encountered by probes in the other two conditions. A qualitative comparison ofvariance in the MSDs in Fig. 3 further suggests a higher degree of structural heterogeneity in the fibroblast (-) group relative to other conditions.

We further analyzed the distribution of maximum displacements of all probes tracked in each condition (Fig. 4). A model-basedclustering analysis was performed on data from the fibroblast (-) condition in order to identify an approximate threshold value of $1.72 \mu \mathrm{m}^{2}$ defining "caged" and "uncaged" probe trajectories across the three conditions compared. In the fibroblast (-) condition, a significant proportion of probe trajectories has displacements above value, while probes in fibroblast (+) are consistently caged (Fig. 4). This result further supports the conclusion from Fig. 3 that the fibroblast (-) cultureshave more heterogeneous ECM microstructure. The distribution of maximum probe displacements in the fibroblast $(-)$ condition contains a visibly bimodal population of tracer probes: a subpopulation of caged probes which resembles those in the control condition, and a subpopulation of uncaged probes which are likely sampling mesoscopic water-filled pockets in which ECM is highly degraded by proteolytic activity of embedded cancer cells.

Additionally, the GSER was applied to particle trajectory data to estimate average local frequencydependent shear moduli for each of the six culture conditions studied. These conditions consisted of the three previously detailed conditions of fibroblast $(+)$, fibroblast $(-)$, and cell-free control in addition

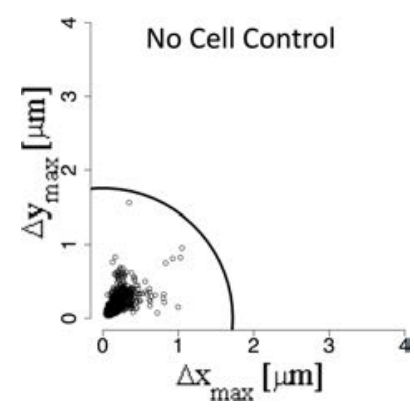

(a)

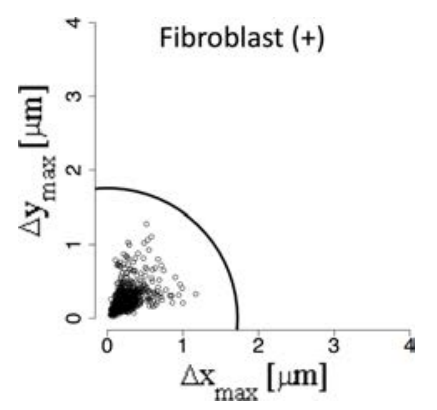

(b)

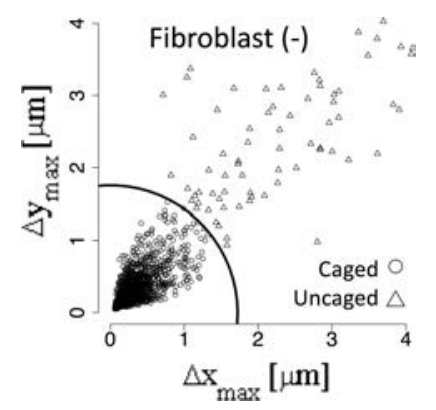

(c)

Fig. 4. Maximum displacement was recorded for all probes within a representative sample well for each of three conditions: (a) no cell control, (b) PANC1 and MRC-5 co-culture, and (c) PANC1 homoculture. The quarter circle at $1.72 \mu \mathrm{m}$ represents a threshold calculated from a clustering algorithmto quantitatively differentiate "caged" and "uncaged" probe trajectories. Average maximum probe displacement compared across conditions (d) further highlights their differences while the standard deviation of the mean seen here as error bars, underlines the large variation in the fibroblast $(-)$ data. 


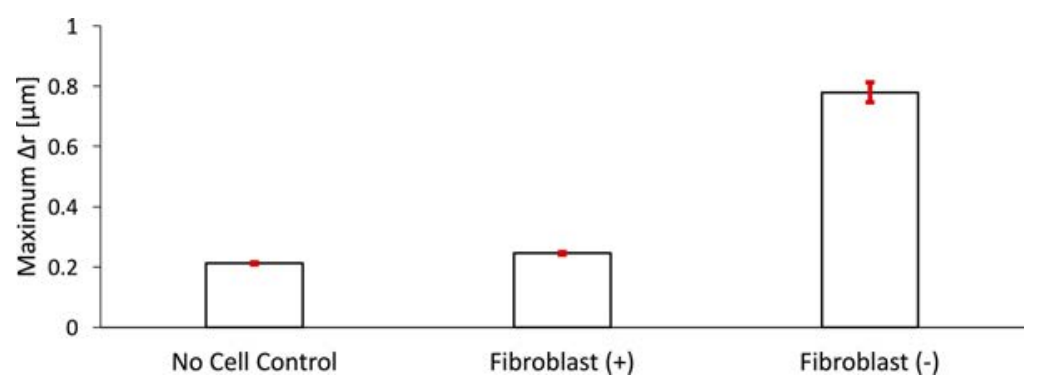

(d)

Fig. 4. (Continued)

to an overlaid PANC1 culture with embedded fibroblasts, an overlaid PANC1 culture without embedded fibroblasts, and a culture with embedded fibroblasts only (no cancer cells). In each case, trajectories from video data acquired at 12 spatial positions were grouped to obtain MSD for computation of complex shear modulus. The value of $G^{\prime}$ at a fixed frequency of $10 \mathrm{~Hz}$ is shown to compare all conditions in a single plot in a Fig. 5. Shear modulus is decreased significantly in the fibroblast $(-)$ condition, but somewhat rescued when fibroblasts are present in addition to cancer cells (the fibroblast $(+)$ condition), coming close to levels displayed in the control samples. Embedded PANC1 and embedded co-culture conditions display a de- crease in $G^{\prime}$ of approximately five-fold compared to all other conditions. This trend is generally consistent with the analysis of probe trajectory heterogeneity, showing an overall degradation of ECM microstructure and rigidity when cancer cells (without fibroblasts) are embedded. The trend in stiffness between the embedded PANC1 and embedded co-culture conditions is mirrored, albeit modestly, in the two overlaid PANC1 counterpart conditions. Frequencydependent data for both storage and loss moduli reveal a similar contrast between the fibroblast $(-)$ condition and all other samples (Fig. 5). Less pronounced, but still potentially meaningful variation exists between the other five conditions when comparing full, frequency-dependent curves.

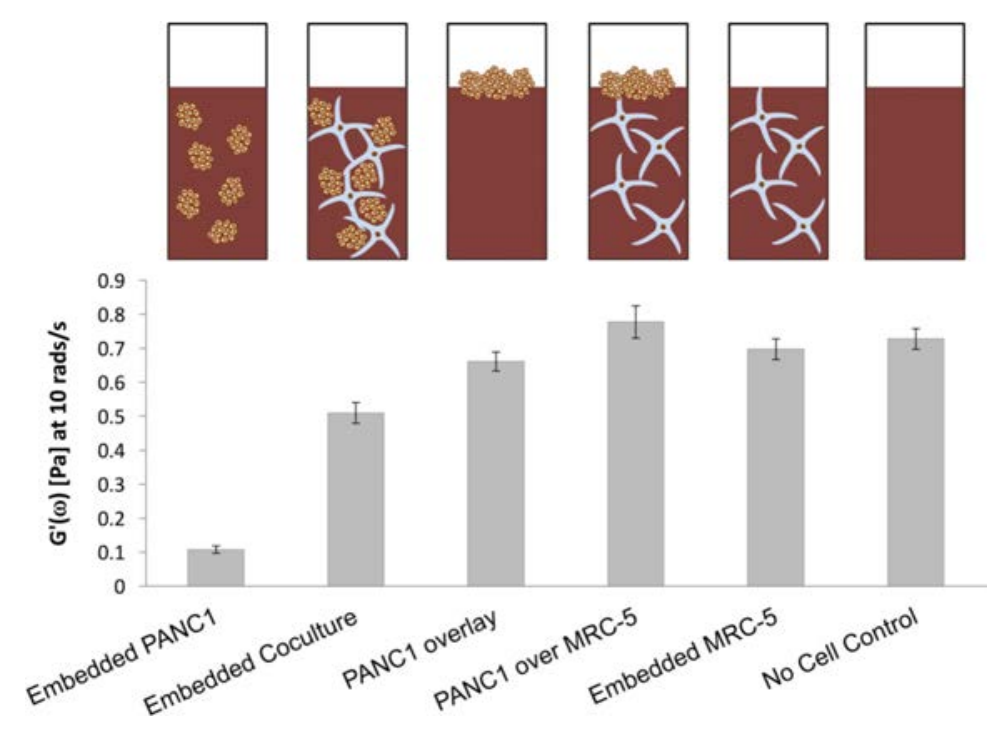

(a)

Fig. 5. (a) Comparison of the real component of the complex shear modulus, $G^{\prime}$, measured in each condition, at $10 \mathrm{~Hz}$. The embedded PANC1 homoculture (no fibroblasts) samples display significantly lower shear modulus relative to other co-culture conditions and geometries. (b) Plots estimated from GSER of the full frequency dependence of the storage $\left(G^{\prime}\right.$, filled circles) and loss $(G$ ", empty circles) moduli for each of the six different culture conditions. 

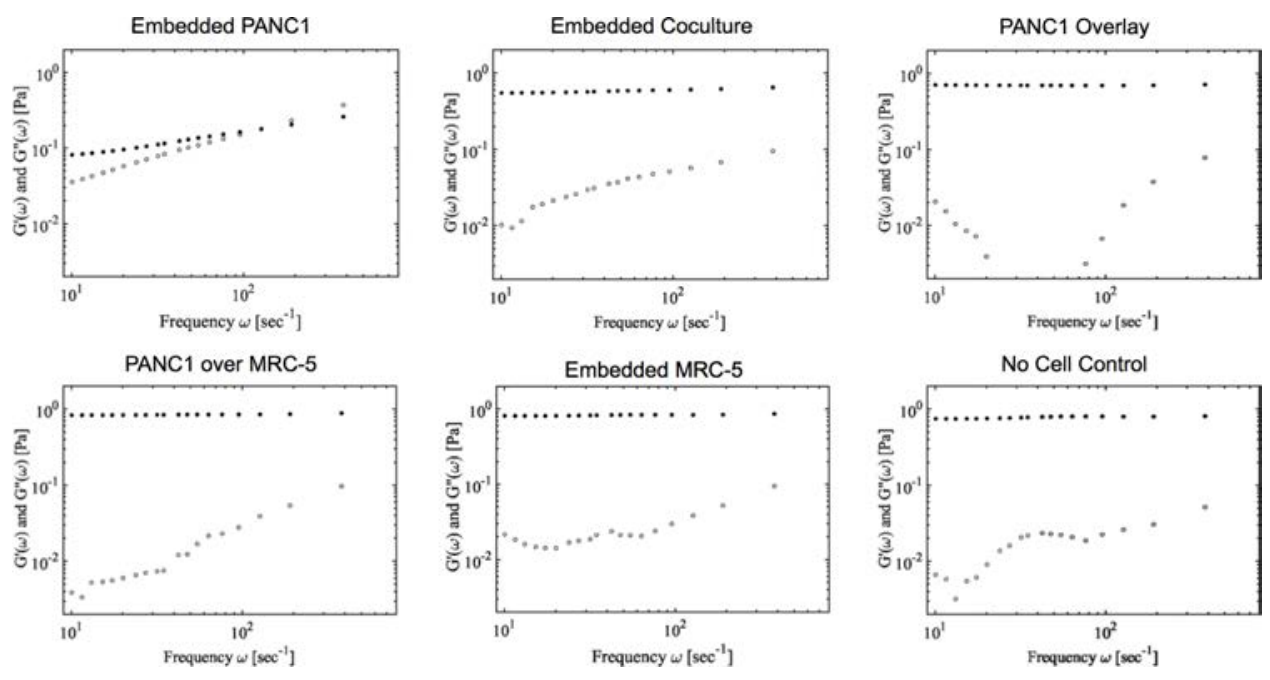

(b)

Fig. 5. (Continued)

\section{Discussion}

While role of paracrine stromal crosstalk in regulating tumor progression has been studied extensively, the complex role of mechanical crosstalk is just beginning to be more fully appreciated. As shown here, the geometry of $3 \mathrm{D}$ cultures as well as the presence or absence of stromal partners leads to significant quantitative changes in both sample microheterogeneity as well as mean overall ECM rigidity. There are multiple factors that could be contributing to these cellular composition and geometry-dependent changes. The ability of PDAC cells to achieve motility within ECM is enabled in part by secretion of proteolytic enzymes including matrix metalloproteases, presumably leading to the observed structural degradation and decreased elasticity of ECM in embedded 3D cultures observed here. As such, 3D cultures of PDAC cells dramatically degrade the ECM when embedded within, but not when the same cells are overlaid upon the surface of the ECM, suggesting that direct $3 \mathrm{D}$ contact is required to initiate proteolytic degradation of the matrix. Furthermore, when PDAC cells are embedded within ECM along with fibroblasts, the latter appears to rescue microstructure and rigidity to values that are comparable, within the sensitivity of this measurement, to cell-free ECM. This can be attributed in part to the ability of fibroblasts to deposit and crosslink ECM, an expected function of this cell type. A logical extension of this particle-tracking study could include biochemical analysis of ECM composition and direct visualization of collagen architecture using second harmonic generation (SHG) imaging. The presence of fibroblasts in co-culture may also increase compressive stress by exerting contractile force on focal adhesion contacts with ECM fibers, which could in turn contribute to compression of ECM pores and uniform suppression of probe mobility. The latter contribution could be further studied via measurements of solid stress in each sample. ${ }^{34}$ In future studies, the role of traction forces could also be measured using timelapse imaging to look atprobe displacements or spatial changes in probe density across longer timescales. Building on this work, these physical changes in ECM architecture could also be measured at multiple time points as the particle tracking is nondestructive to the sample. It is worth noting that although very little change in rheology was observed over five days in the samples studied here, the fact that it was possible to obtain multiple measurements of each sample over that time frame could be important for future applications.

PTM measurements however do have inherent limitations for this set of applications. Passive PTM is only able to provide quantitative shear modulus estimates for relatively soft materials, limited ultimately by spatial resolution and the ability of subpixel tracking to resolve small displacements of probes in stiff materials. PTM is also restricted to a 
range of frequencies for which the GSER is valid. ${ }^{35}$ The necessary introduction of tracer probes, and the need for a clear and steady microscopic video tether this technique to controllable in vitro models. As such, the sample dimensions are bound by both biological limits (i.e., developmental restraints set by synthetic growth conditions) and optical constraints (i.e., limiting the thickness of the sample to allow for high resolution imaging). Finally, one of the strengths of the method lies in the statistics of monitoring the trajectories of many individual probes, but the need to analyze regions containing many probes also limits the spatial resolution of the output. While this is adequate for purposes of the present study, which measures rheology at a given position within a larger sample, but studies that set out to measure rheology on a smaller scale, for example, a local change at the leading edge of a single cell, may require the use of active techniques such as optical tweezers. Besides the inherent limitations of the measurement, the roles of in vivo biophysical influences including interstitial pressure and fluid flow through blood and lymphatic vessels in the tumor are absent from the 3D tumor models used in this study.

Even in relatively simple in vitro tumor model systems, the ability to quantify dynamic changes in rheology could provide useful insight into how cancer therapeutics impact upon a tumor's mechanical microenvironment. The methodology developed in this study could potentially be applied for analysis of response to treatment strategies that are intended to act upon the stroma itself and/or mechanisms of tumor-promoting stromal crosstalk. ${ }^{36-38}$ Indeed for tumors of the pancreas, stromal depletion therapies have been developed to target the stroma as a physical barrier to drug perfusion and enhance delivery of subsequent therapeutics. ${ }^{36,37}$ In this context, however, the importance of being able to measure intervention-dependent changes in stromal mechanics is underscored by studies that highlight the complex role of the stroma, both constraining and promoting pancreatic tumor progression. ${ }^{39,40}$ In view of this application, the methodology presented here could be a powerful component of an in vitro screening tool to report therapeutic impact upon the mechanical microenvironment. This approach is inherently conducive to other imaging-based screening approaches that provide quantitative assessment of cytotoxic response of 3D cell cultures ${ }^{41}$ potentially allowing for high-content, co-registered evaluation of the impact of therapeutics on tumors as well as (components of) their microenvironment.

\section{Acknowledgments}

We gratefully acknowledge funding from the National Cancer Institute (R00 CA155045, PI Celli) which has supported this work. We are also grateful to Dr. Imran Rizvi of Massachusetts General Hospital for many helpful and productive conversations relevant to this study.

\section{References}

1. O. Trédan, C. M. Galmarini, K. Patel, I. F. Tannock, "Drug resistance and the solid tumor microenvironment," J. Nat. Cancer Inst. 99, 1441-1454 (2007).

2. F. Mbeunkui, D. J. Johann, "Cancer and the tumor microenvironment: A review of an essential relationship," Cancer Chemother. Pharmacol. 63, 571-582 (2009).

3. D. T. Butcher, T. Alliston, V. M. Weaver, "A tense situation: Forcing tumour progression," Nat. Rev. Cancer 9, 108-122 (2009).

4. J. P. Celli, M. D. Anderson, Reference Module in Life Sciences, Elsevier (2017), doi: 10.1016/B978-012-809633-8.12037-0.

5. A. Vonlaufen et al., "Pancreatic stellate cells and pancreatic cancer cells: An unholy alliance," Cancer Res. 68, 7707-7710 (2008).

6. R. Kalluri, M. Zeisberg, "Fibroblasts in cancer," Nat. Rev. Cancer 6, 392-401 (2006).

7. J. A. Tuxhorn et al., "Reactive stroma in human prostate cancer," Clin. Cancer Res. 8, 2912-2923 (2002).

8. K. Alessandri et al., "Cellular capsules as a tool for multicellular spheroid production and for investigating the mechanics of tumor progression in vitro," Proc. Nat. Acad. Sci. 110, 14843-14848 (2013).

9. S. Kumar, V. M. Weaver, "Mechanics, malignancy, and metastasis: The force journey of a tumor cell," Cancer Metastasis Rev 28, 113-127 (2009).

10. M. J. Paszek et al., "Tensional homeostasis and the malignant phenotype," Cancer Cell 8, 241-254 (2005).

11. J. Mollenhauer, I. Roether, H. F. Kern, "Distribution of extracellular matrix proteins in pancreatic ductal adenocarcinoma and its influence on tumor cell proliferation in vitro," Pancreas 2, 14-24 (1987).

12. D. Mahadevan, D. D. Von Hoff, "Tumor-stroma interactions in pancreatic ductal adenocarcinoma," Mol. Cancer Ther. 6, 1186-1197 (2007). 
13. R. A. Walker, "The complexities of breast cancer desmoplasi," Breast Cancer Res. 3, 143-145 (2001).

14. J. Debnath, J. S. Brugge, "Modeling glandular epithelial cancers in three-dimensional cultures," Nat. Rev. Cancer 5, 675-688 (2005).

15. J. Debnath, S. K. Muthuswamy, J. S. Brugge, "Morphogenesis and oncogenesis of MCF-10A mammary epithelial acini grown in three-dimensional basement membrane cultures," Methods $\mathbf{3 0}$, 256-268 (2003).

16. M. J. Bissell, D. Radisky, "Putting tumours in context," Nat Rev Cancer 1, 46-54 (2001).

17. G. Y. Lee, P. A. Kenny, E. H. Lee, M. J. Bissell, "Three-dimensional culture models of normal and malignant breast epithelial cells," Nat. Methods 4, 359-365 (2007).

18. C. M. Nelson, M. J. Bissell, "Of extracellular matrix, scaffolds, and signaling: Tissue architecture regulates development, homeostasis, and cancer," Annu. Rev. Cell. Dev. Biol. 22, 287-309 (2006).

19. B. Grun et al., "Three-dimensional in vitro cell biology models of ovarian and endometrial cancer," Cell Proliferat. 42, 219-228 (2009).

20. M. J. Bissell et al., "Tissue structure, nuclear organization, and gene expression in normal and malignant breast," Cancer Res. 59, 1757s-1763s (1999).

21. T. A. Ulrich, A. Jain, K. Tanner, J. L. MacKay, S. Kumar, "Probing cellular mechanobiology in threedimensional culture with collagen-agarose matrices," Biomaterials 31, 1875-1884 (2010).

22. A. O. Abu-Yousif, I. Rizvi, C. L. Evans, J. P. Celli, T. Hasan, "PuraMatrix encapsulation of cancer cells," J. Vis. Exp. 34, e1692 (2009).

23. D. Wirtz, K. Konstantopoulos, P. C. Searson, "The physics of cancer: The role of physical interactions and mechanical forces in metastasis," Nat. Rev. Cancer 11, 512-522 (2011).

24. M. T. Valentine et al., "Investigating the microenvironments of inhomogeneous soft materials with multiple particle tracking," Phys. Rev. E64, 061506 (2001).

25. T. G. Mason, K. Ganesan, J. H. van Zanten, D. Wirtz, S. C. Kuo, "Particle tracking microrheology of complex fluids," Phys. Rev. Lett. 79, 3282-3285 (1997).

26. T. G. Mason, D. A. Weitz, "Optical measurements of frequency-dependent linear viscoelastic moduli of complex fluids," Phys. Rev. Lett. 74, 1250 (1995).

27. F. Zvietcovich, J. P. Rolland, J. Yao, P. Meemon, K. J. Parker, "Comparative study of shear wave-based elastography techniques in optical coherence tomography," J. Biomed. Opt. 22, 035010-035010 (2017).

28. D. Wirtz, "Particle-tracking microrheology of living cells: Principles and applications," Annu. Rev. Biophys. 38, 301-326 (2009).

29. D. P. Jones, W. Hanna, H. El-Hamidi, J. P. Celli, "Longitudinal measurement of extracellular matrix rigidity in $3 \mathrm{D}$ tumor models using particle-tracking microrheology," J. Vis. Exp. 88, e51302 (2014).

30. M. Makale, "Cellular mechanobiology and cancer metastasis," Birth Defects Res. C Embryo Today Rev. 81, 329-343 (2007).

31. D. P. Jones, W. Hanna, J. P. Celli, "Mapping dynamic mechanical remodeling in $3 \mathrm{D}$ tumor models via particle tracking microrheology,"SPIE BiOS, pp. 93270L-93275L, International Society for Optics and Photonics (2015), doi: 10.1117/12.2084282.

32. N. G. V. Pelletier, P. Fournier, M. L. Kilfoil, "Microrheology of microtubule solutions and actinmicrotubule composite networks," Phys. Rev. Lett. 102, 1-4 (2009).

33. C. Fraley, A. E. Raftery, "Model-based methods of classification: Using the mclust software in chemometrics," J. Stat. Software 18, 1-13 (2007).

34. M. D. Nieskoski, J. Gunn, K. Marra, B. S. Trembly, B. W. Pogue, "Pancreas tumor interstitial pressure catheter measurement," SPIE BiOS, pp. 969408 969407, International Society for Optics and Photonics (2016), doi: 10.1117/12.2214181.

35. A. J. Levine, T. C. Lubensky, "One- and two-particle microrheology," Phys. Rev. Lett. 85, 1774 (2000).

36. K. Garber, Oxford University Press (2010).

37. K. P. Olive et al., "Inhibition of Hedgehog signaling enhances delivery of chemotherapy in a mouse model of pancreatic cancer," Science 324, 14571461 (2009).

38. J. P. Celli, "Stromal interactions as regulators of tumor growth and therapeutic response: A potential target for photodynamic therapy?" Isr. J. Chem. 52, 757-766 (2012).

39. B. C. Özdemir et al., "Depletion of carcinoma-associated fibroblasts and fibrosis induces immunosuppression and accelerates pancreas cancer with reduced survival," Cancer Cell 25, 719-734 (2014).

40. E. W. Roberts et al., "Depletion of stromal cells expressing fibroblast activation protein- $\alpha$ from skeletal muscle and bone marrow results in cachexia and anemia," J. Exp. Med. 210, 1137-1151 (2013).

41. J. P. Celli et al., "An imaging-based platform for high-content, quantitative evaluation of therapeutic response in 3D tumour models," Sci. Rep. 4, 3751 (2014). 